\title{
High-Sensitivity Visible-Blind AlGaN Photodiodes and Photodiode Arrays
}

J.D. Brown, J. Matthews, S. Harney, J. Boney, and J.F. Schetzina

Department of Physics - Box 8202

North Carolina State University

Raleigh, NC 27695-8202

J.D. Benson and K.V. Dang

Night Vision and Electronic Sensors Directorate

10221 Burbick Rd, St. 430

Fort Belvoir, VA 22060, USA.

Thomas Nohava, Wei Yang, and Subash Krishnankutty

Honeywell Technology Center

12001 State Highway

Plymouth, Minnesota 55441.

\section{ABSTRACT}

Visible-blind UV cameras based on a 32 × 32 array of backside-illuminated GaN/AlGaN p-i-n photodiodes have been successfully demonstrated. The photodiode arrays were hybridized to silicon readout integrated circuits (ROICs) using In bump bonds. Output from the UV cameras were recorded at room temperature at frame rates of 30-240 Hz. These new visible-blind digital cameras are sensitive to radiation from 285$365 \mathrm{~nm}$ in the UV spectral region.

\section{INTRODUCTION}

The III-V nitrides have developed rapidly over the past five years. This has lead to the commercialization of blue and green light emitting diodes, along with demonstrations of violet laser diodes and a variety of electronic devices [1-2]. In addition, a number of photodetectors based on photoconductive elements and arrays [3-6] along with junction devices have also recently been reported [7-9].

This paper reports new images from the first successful demonstration of ultraviolet (UV) digital cameras [8] based on an array of GaN/AlGaN heterostructure p-i-n photodiodes. These new ( $32 \times 32$ pixel) digital imagers are designed to sense radiation in the 285-365 nm wavelength band in the UV spectral region. Thus, the digital camera is visible-blind but is not solar-blind $(250-280 \mathrm{~nm})$. A discussion of photodiode properties is followed by a description of the experimental procedures employed to synthesize, process and study discrete photodiodes and photodiode arrays, and then by the experimental results obtained.

\section{EXPERIMENTAL DETAILS}

The photodiode structure employed in the present work is shown schematically in Figure 1. It consists of a base layer of $\mathrm{n}-\mathrm{AlGaN}(\sim 20 \% \mathrm{Al})$ followed by an undoped $\mathrm{GaN}$ layer and a p-GaN layer. The photodiode structure is deposited by MOVPE onto a 


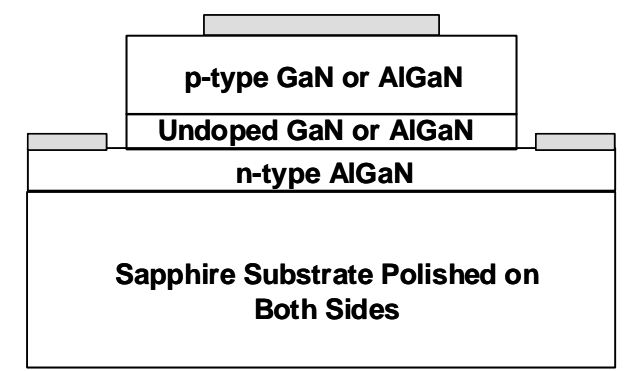

Figure 1 Schematic of p-i-n photodiode structure.

polished sapphire wafer to permit illumination of the device through the substrate. The photodiode structure employed in our initial experiments responds to UV light in the wavelength band from about $320 \mathrm{~nm}$ to $365 \mathrm{~nm}$. At wavelengths shorter than $320 \mathrm{~nm}$, the incoming light is absorbed in the thick $\mathrm{AlGaN}$ base layer $(20 \% \mathrm{Al})$ and the junction is not illuminated. Likewise, the diode does not respond to wavelengths greater than 365 $\mathrm{nm}$, since this corresponds to the optical absorption edge of $\mathrm{GaN}$ at $300 \mathrm{~K}$. By increasing the $\mathrm{Al}$ content of the base layer it is possible to increase the optical bandwidth of the diode's UV responsivity. Likewise, by adding Al to the top layers, it is possible to change the diode UV responsivity band to other wavelength regions in the UV. Thus, UV detectors that sense different UV "colors" are possible.

Diode structures of the type shown were prepared by MOVPE both at North Carolina State University (NCSU) and at the Honeywell Technology Center using low-pressure, vertical-flow MOVPE reactors that employ high speed substrate rotation during film growth. The photodiode structures were deposited onto 2 in diameter c-plane sapphire substrates. The growth was initiated by depositing a thin AlN buffer layer at $500-650{ }^{\circ} \mathrm{C}$; all subsequent layers were grown at $1050-1080{ }^{\circ} \mathrm{C}$.

All device processing was completed at NCSU using standard semiconductor processing techniques which included photolithography using appropriately-designed masks, reactive ion etching to define mesa structures, and metallizations to provide ohmic contacts to the n-type and p-type layers of the device.

Spectral responsivity measurements [8] were completed at NCSU on selected discrete photodiodes. The $300 \mathrm{~K}$ dynamic resistance of the photodiode at zero-bias, $\mathrm{R}_{0}$, was measured for selected devices using a shielded low-noise enclosure and shielded probe tips. These measurements were combined with the device area $A$ to obtain the $R_{0} A$ product which was then used to obtain an estimate of the detector detectivity $\mathrm{D}^{*}$ [8]. In order to use the GaN/AlGaN photodiodes as the basis for a new visible-blind UV digital camera it is necessary to employ flip-chip bonding techniques to hybridize an appropriately sized photodiode array to a silicon readout integrated circuit (ROIC). In the present case, NCSU designed and purchased a mask set for a photodiode array that matched a 32 x 32 ROIC chip provided by the Night Vision Laboratory (NVL) at Ft. Belvoir. Prior to hybridization at NVL, In bumps were deposited onto each of the mesas and n-contact layers of the photodiode array and onto the corresponding areas of the ROIC using NCSU facilities. Each $32 \times 32 \mathrm{GaN} / \mathrm{AlGaN}$ photodiode array was hybridized to the silicon ROIC using facilities at NVL and then cemented onto a leadless 
chip carrier. Gold wire bonds were then used to link the various input/output channels from the ROIC to the leadless chip carrier. A closeup of a hybridized UV photodiode array is shown in Figure 2 mounted onto a printed circuit board in preparation for testing.

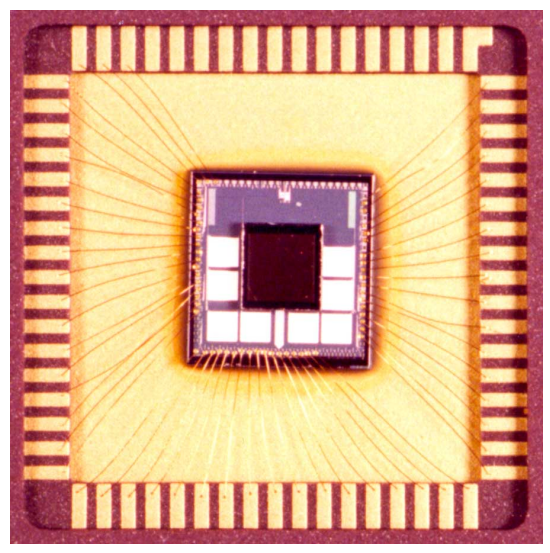

Figure 2 Photograph of a hybridized UV focal plane array(FPA). The dark square in the middle is the GaN/AlGaN photodiode array which is In bump bonded to the larger ROIC chip. Wire bonds connect the ROIC outputs to the leadless chip carrier.

The experimental setup used for testing the GaN/AlGaN UV FPAs is shown in Figure 3 below. It consists of an alphanumeric UV source, a quartz focusing lens, the UV digital

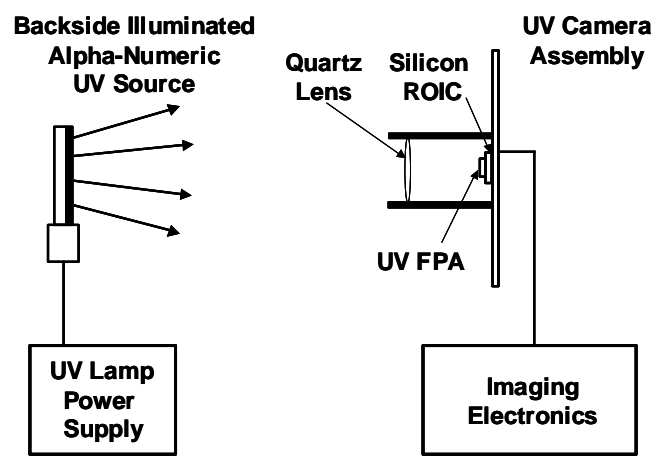

Figure 3 Experimental setup for testing the UV digital camera.

camera, and a computerized imaging system [8]. A UV Hg lamp fitted with a fluorescent filter was used as a UV light source. This produced a UV output centered at about $350 \mathrm{~nm}$ with a FWHM of $\sim 40 \mathrm{~nm}$. A computer-generated template was then attached to the front of the UV source generating a UV back-lighted scene. A fused quartz lens of focal length $25 \mathrm{~mm}$ was used to focus the UV scene onto the AlGaN FPA as shown in Figure 3. 


\section{RESULTS AND DISCUSSION}

The room temperature spectral responsivity of two discrete $\mathrm{AlGaN}$ p-i-n photodiodes is shown in Figure 4. The first device consists of a base n-type layer of $\mathrm{Al}_{0.2} \mathrm{Ga}_{0.8} \mathrm{~N}$, an undoped GaN layer, and a p-type GaN:Mg layer. The device has a sharp cut-on beginning at about $365 \mathrm{~nm}$, which corresponds to the optical absorption edge of $\mathrm{GaN}$ at room temperature. The responsivity reaches its maximum value of $0.2 \mathrm{~A} / \mathrm{W}$ at a wavelength of $358 \mathrm{~nm}$, corresponding to an internal quantum efficiency of $\sim 82 \%$ [8]. $\mathrm{D}^{*}=6.3 \times 10^{13} \mathrm{~cm} \mathrm{~Hz}^{1 / 2} \mathrm{~W}^{-1}$ for this type of UV photodiode. This is one of the largest $\mathrm{D}^{*}$ values ever obtained for any semiconductor photodiode at any temperature.
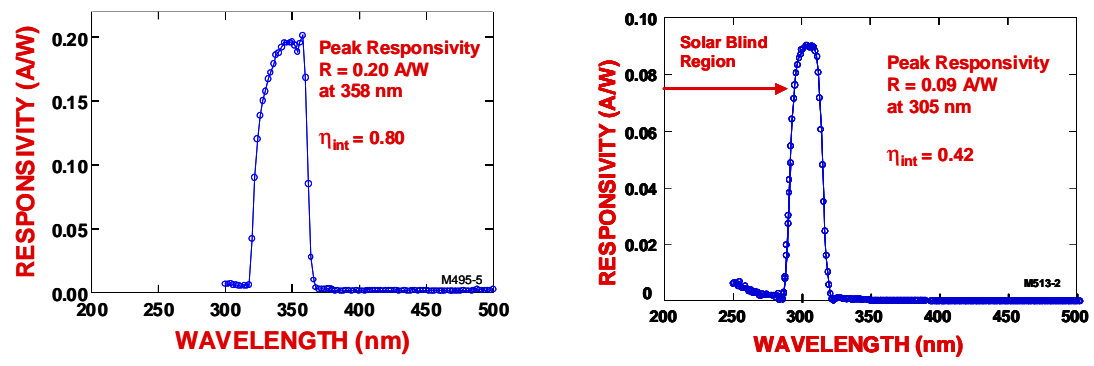

Figure 4. Spectral responsivities for two different p-i-n photodiodes.

The second device consists of a base n-type layer of $\mathrm{Al}_{0.33} \mathrm{Ga}_{0.67} \mathrm{~N}$, an undoped $\mathrm{Al}_{0.16} \mathrm{Ga}_{0.84} \mathrm{~N}$ layer, and a $\mathrm{p}$-type $\mathrm{Al}_{0.16} \mathrm{Ga}_{0.84} \mathrm{~N}$ layer. This photodiode has a sharp cut on at $\sim 320 \mathrm{~nm}$ and displays a peak responsivity of $0.09 \mathrm{~A} / \mathrm{W}$ at $305 \mathrm{~nm}$, corresponding an internal quantum efficiency of $\sim 42 \%$. Its responsivity band covers the region 285-320 $\mathrm{nm} . \mathrm{D}^{*}=2.1 \times 10^{13} \mathrm{~cm} \mathrm{~Hz}^{1 / 2} \mathrm{~W}^{-1}$ for this type of AlGaN heterostructure $\mathrm{p}$-i-n photodiode.

Photodiode arrays have been successfully fabricated and tested for both of these types of devices. In addition, we have subjected selected devices to light pulses from a xenon lamp and have measured their transient response using an oscilloscope. From these measurements we estimate that the risetime of the nitride photodiodes is about $1 \mathrm{~ns}$ and the falltime is less that $1 \mu \mathrm{s}$. Thus, they are quite suitable for UV imaging applications.

Initial images from the $32 \times 32$ UV digital camera have already been reported [8] and will not be reproduced here. Rather, we will report new images obtained from several new UV scenes. The first set of UV images obtained from one of the $32 \times 32 \mathrm{UV}$ camera chips is shown below in Figure 5.
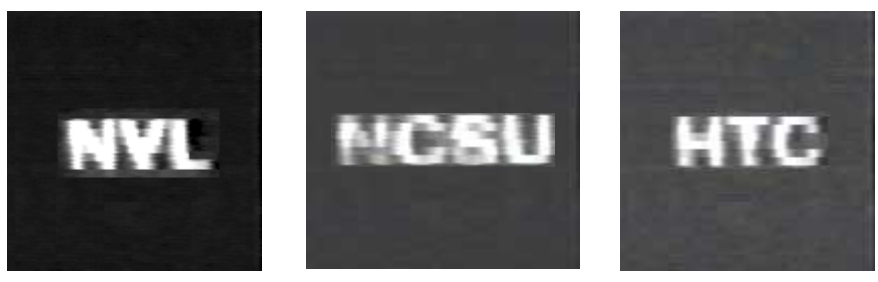

Figure 5. Alpha/Numeric images from visible-blind UV digital camera. 

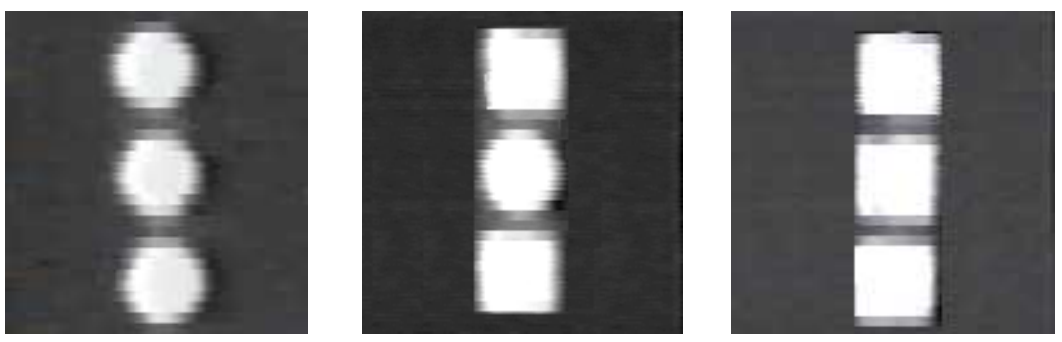

Figure 6 UV images of selected geometric shapes.

In Figure 6, UV images of several sets of geometric objects are shown. It is seen that the squares image reasonably well, but the circles suffer from the coarseness of the $32 \times 32$ pixel format.

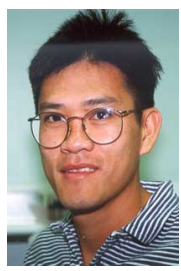

Visible Image

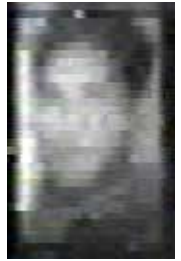

UV Image

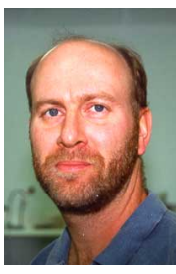

Visible Image

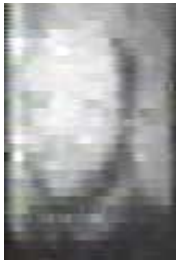

UV Image

Figure 7 UV images of Dr. Dang (left) and Dr. Benson (right) of NVL

Figure 7 shows images of Drs. Dang and Benson, the UV images are produced with the back-lighting method described earlier. The quality of the UV images are similar to those obtainable from $32 \times 32$ pixel arrays of silicon photodiodes. Although the image resolution would greatly improve with the use of UV imagers based on larger 128x128 arrays of photodiodes, the two subjects are readily discernable using the $32 \times 32$ arrays.

\section{SUMMARY AND CONCLUSIONS}

These UV camera demonstrations represent another historical advancement in optoelectronic devices based on III-V nitride materials. When fully developed into large- 
format photodiode arrays, this new type of UV digital camera may be used in many wideranging applications including biological agent detection, missile and shellfire detection, atmospheric ozone-level detection, welding imagery, and flame sensing. In addition, large-format UV staring FPAs based on nitride photodiodes may play an important role in obtaining UV images of the stars and other astronomical objects of importance in understanding the creation and evolution of the universe.

\section{ACKNOWLEDGMENTS}

The work at NCSU is being supported by Army Research Office grants DAAH04-951-0627 and DAAD19-99-0113 administered by Dr. M. Dutta and by a Defense Advanced Research Projects Agency grant DAAD19-99-0010 under the Solar Blind Detector Program directed by Dr. R. Leheney. Honeywell would like to acknowledge the support and encouragement of Dr. Paul Schreiber at the Air Force Research Laboratories, WrightPatterson Air ForceBase, Ohio.

\section{REFERENCES}

1. S. Nakamura, T. Mukai, M. Senoh, App. Phys. Lett. 64, 1687 (1994).

2. Nakamura, G. Fasol, The Blue Laser Diode, published by SpringerVerlag (Heidelberg), 0 (1997). ISBN 3-540-61590-3, 343 pages.

3. Dennis K. Wickenden, Zhenchun Huang, D. Brent Mott, and Peter K. Shu, Johns Hopkins APL Technical Digest, 18(2) 217 (1997).

4. Z.C. Huang, D.B. Mott, P.K. Shu, CP420, Space Technology and Applications International Forum-1998, American Institute of Physics Conference Proceedings 1-56396-747 (1998).

5. T.Z.C. Huang, D.B. Mott, A. La, paper presented at SPIE Conference, Denver (July, 1999), to be published in SPIE conference proceedings.

6. Hadis Morkoc, Naval Research Reviews 51, 24 (1999). This recent review article can be accessed on the World Wide Web at http://www.onr.navy.mil/onr/pubs.htm. It contains many photodetector references.

7. Wei Yang, Thomas Novova, Subash Krishnankutty, Robert Torreano, Scott McPherson, Holly Marsh, Appl. Phys. Lett. 73(8), 1086 (1998).

8. J.D. Brown, Zhonghai Yu, J. Matthews, S. Harney, J. Boney, J.F. Schetzina, J.D. Benson, K.W. Dang, C. Terrill, Thomas Nohava, Wei Yang, Subash Krishnankutty, MRS Internet J. Nitride Semicond. Res. 4, 9(1999).

9. K. Linthicum, D. Hanser, T. Zheleva, O.K. Nam, R.F. Davis, Naval Research Reviews 51, 44 (1999). This review paper on ELO and pendio-epitaxy can be accessed on the World Wide Web at http://www.onr.navy.mil/onr/pubs.htm. 